\title{
Genetic characterization of Toxoplasma gondii in Yunnan black goats (Capra hircus) in southwest China by PCR-RFLP
}

Qiang Miao ${ }^{1,2+}$, Si-Yang Huang ${ }^{2 \dagger}$, Si-Yuan Qin ${ }^{2,3}$, Xin Yu ${ }^{1}$, Yan Yang ${ }^{1}$, Jian-Fa Yang ${ }^{1}$, Xing-Quan Zhu ${ }^{1,2}$ and Feng-Cai Zou ${ }^{1 *}$

\begin{abstract}
Background: Toxoplasma gondii is a protozoan parasite that infects almost all warm-blooded animals and human beings. Goats are one of the susceptible animals to T. gondii. However, little is known of genetic diversity of T. gondii in Yunnan black goats in China. The objective of this present study was to determine the genotypes of T. gondii isolates from black goats in Yunnan province, southwest China.

Methods: Genomic DNA was extracted from liver $(n=403)$, lung $(n=403)$ and lymph nodes $(n=250)$ of Yunnan black goats and assayed for T. gondii infection by semi-nested PCR of B1 gene. Then, the positive DNA samples were typed at 10 genetic markers using polymerase chain reaction-restriction fragment length polymorphism (PCR-RFLP) technology. These markers include 9 nuclear loci, namely, SAG1, SAG2 (5'-SAG2 and 3'-SAG2, alternative SAG2), SAG3, BTUB, GRA6, c22-8, c29-2, L358, PK1, and an apicoplast locus Apico.

Results: Out of 403 tested samples, 20 (4.96\%) DNA samples were T. gondii positive by amplification of B1 gene. Among them, 2 isolates were genotyped at all loci, and 6 isolates were genotyped for 8 or more loci. In total, seven samples belong to ToxoDB PCR-RFLP genotype\#10 (Type I), and one belongs to genotype ToxoDB \#9.

Conclusions: To our knowledge, this is the first report of ToxoDB\#9 and ToxoDB\#10 T. gondii in Yunnan black goats in China. These results revealed a wide distribution of these T. gondii in Yunnan black goats in China, which has important implications for public health.
\end{abstract}

Keywords: Toxoplasma gondii, Genotype, PCR-RFLP, Yunnan black goats, China

\section{Background}

Toxoplasma gondii is an obligate intracellular parasite, causing toxoplasmosis in almost all warm-blooded animals and humans [1]. Generally, T. gondii infection rarely causes clinical symptoms in healthy individuals, however, it can cause severe diseases, even fatal to AIDS patients or those individuals with cancer undergoing immuno-suppressive therapy [2].

Yunnan is a province having 25 different ethnic groups, where halal food like mutton is well-received for human consumption. Goats are commonly infected with $T$. gondii [1], and it can be a potential source for human toxoplasmosis through consumption of uncooked or raw mutton

\footnotetext{
* Correspondence: zfc1207@vip.163.com

${ }^{\dagger}$ Equal contributors

${ }^{1}$ College of Animal Science and Technology, Yunnan Agricultural University, Kunming, Yunnan Province 650201, PR China

Full list of author information is available at the end of the article
}

containing T. gondii tissue cysts [3]. In view of previous serology reports in Yunnan Province, seroprevalence of $T$. gondii infection was $21.6 \%$ [4], 17.0\% [5], 27.1\% [6], 12.6\% [7], 19.9\% [8], 6.3\% [9] in pet dogs, pigs, equids, peafowls, black-headed gulls and goats, respectively, which revealed a widely distribution of $T$. gondii infection in this province. In addition, variable genotypes of $T$. gondii were identified from HIV positive patients [10], pigs [11], cats [12] and bats [13] in Yunnan Province. However, little information is available about the genetic characterization of $T$. gondii in Yunnan black goats in China. Thus, the objective of this present study was to determine the genotypes of $T$. gondii isolated from black goats in Yunnan province, southwest China, and the results would provide fundamental data for prevention and control of $T$. gondii infection in black goats. 


\section{Methods}

\section{Ethics statement}

The collection of tissue samples from Yunnan black goats in this study was agreed by the abattoir manager. All animals were handled in strict accordance with good animal practice according to the Animal Ethics Procedures and Guidelines of the People's Republic of China.

\section{Sample collection}

In total, liver, lung and lymph nodes from 403 Yunnan black goats were collected randomly from different administrative regions in Yunnan province between June 2011 and March 2014, including 103 from Yuxi, 68 from Honghe, 85 from Kunming, 50 from Chuxiong and 97 from Qujing. Then, tissue samples were stored at $-20^{\circ} \mathrm{C}$ prior to use.

\section{Genomic DNA extraction}

Genomic DNA was extracted from different tissues using TIANamp Genomic DNA kit (TianGen ${ }^{\mathrm{Tm}}$, Beijing, China) according to the manufacturer's instructions. In brief, $50 \mathrm{mg}$ of each tissue was treated with sodium dodecyl sulphate $(200 \mu \mathrm{L})$ and proteinase $\mathrm{K}(20 \mu \mathrm{L})$ at $56^{\circ} \mathrm{C}$ for overnight digestion in a thermostat water bath. DNA samples were purified by silica gel column chromatography and obtained with $50 \mu \mathrm{L}$ elution buffer.

\section{Genetic characterization of $T$. gondii isolates}

The DNA samples of Yunnan black goats tissues were first examined for $T$. gondii infection by PCR amplification of B1gene [14] and then the positive samples were genotyped using Multi-locus PCR-RFLP (Mn-PCR-RFLP) method [15]. In brief, the target DNA sequences were amplified by multiplex PCR using external primers for all 10 markers. Then $1 \mu \mathrm{L}$ of the products served as template DNA for nested PCR amplification with internal primers for each marker. The nested PCR products were digested with restriction enzymes for $3 \mathrm{~h}$, at the corresponding temperature for each enzyme following the instruction for each enzyme. The restriction fragments were resolved in 2.5\% agarose gel to display DNA fragment length polymorphism using a gel document system (UVP Gel Doc-It ${ }^{\mathrm{Tm}}$ Imaging System, Cambridge, U.K.).

\section{Statistical analyses}

The prevalence data were analyzed by Chi-squared tests using the program SPSS as previously (Release 19.0 standard version, SPSS Inc., Chicago, Illinois), and the probability $(P)$ value $<0.05$ was considered statistically significant.

\section{Results and discussion}

Out of 403 black goats, twenty (4.96\%) were T. gondii B1 gene positive, and were distributed in all five administrative regions with the prevalence varying from $1.18 \%$ (Kunming) to $10.31 \%$ (Qujing), but the difference was not statistically significant $(P>0.05)$. The prevalence in different tissues ranged from $0.99 \%$ (lung) to $4.40 \%$ (lymph nodes), and the difference was statistically significant $(P<0.05)$ (Table 1$)$. Previously, the overall prevalence of $65.79 \%$ [16] in hilar lymph nodes from pigs in south China, 7.83\% [17] in hilar lymph nodes from pigs in central China, 10.98\% [12] in liver from cats in southwest China, 53.85\% [18] in lung from Microtus fortis in northeastern China were reported. Such significant differences in prevalence in various animals may due to several reasons, such as geographical origin, the tested number and the susceptibility to T. gondii of different animals.

Due to the low DNA concentration, only $2 T$. gondii isolates from Yunnan black goats presented complete genotyping data, and $6 T$. gondii isolates were genotyped at 8 or more loci, whereas the rest $12 T$. gondii isolates were genotyped by less than 6 loci, and considered unreliable, therefore not included for further analysis. Of these $8 \mathrm{~T}$. gondii isolates with reliable typing data, two genotypes were revealed, namelyToxoDB\#9 and ToxoDB\#10 (Type I) (ToxoDB Version 11.0). Both genotypes were identified from Yunnan black goats in China for the first time, and these genotyping results were summarized in Table 2 . Only one genotype (ToxoDB\#9) was identified from Yuxi city in Yunnan province. This genotype has also been identified in previous studies in various animals: cats from Beijing Municipality, Guangdong, Anhui, Yunnan, Guizhou, Shandong, and Hubei provinces [12,19-23]; pigs from Guangdong, Henan, Yunnan and Anhui provinces $[11,17,24,25]$; and bats from Guangxi Zhuang Autonomous Region [13]. Therefore, ToxoDB\#9 is a predominant lineage prevalent in Mainland China. Previous studies showed that ToxoDB\#9 has been isolated from North and

Table 1 Prevalence of Toxoplasma gondii infection in different tissues of Yunnan black goats detected by PCR

\begin{tabular}{|c|c|c|c|c|c|c|c|c|}
\hline \multirow[t]{2}{*}{ Category } & \multicolumn{5}{|c|}{ Regions } & \multicolumn{3}{|c|}{ Tissues } \\
\hline & Yuxi & Honghe & Kunming & Chuxiong & Qujing & Liver & Lung & Lymph nodes \\
\hline Total no. & 103 & 68 & 85 & 50 & 97 & 403 & 403 & 250 \\
\hline Positive no. & 5 & 3 & 1 & 1 & 10 & 5 & 4 & 11 \\
\hline Prevalence (\%) & 4.85 & 4.41 & 1.18 & 2 & 10.31 & 1.24 & 0.99 & 4.40 \\
\hline Total prevalence (\%) & 4.96 & & & & & 1.89 & & \\
\hline
\end{tabular}


Table 2 Summary of genotyping of Toxoplasma gondii isolates from Yunnan black goats in different administrative areas of Yunnan province, southwest China

\begin{tabular}{|c|c|c|c|c|c|c|c|c|c|c|c|c|c|c|c|}
\hline Isolate ID & Host & tissue & Location & SAG1 & $\begin{array}{l}5^{\prime}+3^{\prime} \\
\text { SAG2 }\end{array}$ & $\begin{array}{l}\text { Alternative } \\
\text { SAG2 }\end{array}$ & SAG3 & BTUB & GRA6 & c22-8 & c29-2 & L358 & PK1 & Apico & Genotype \\
\hline GT1 & Goat & & United States & I & 1 & 1 & 1 & I & 1 & 1 & I & 1 & I & I & $\begin{array}{l}\text { Reference, } \\
\text { Type I, } \\
\text { ToxoDB\#10 }\end{array}$ \\
\hline PTG & Sheep & & United States & $\|/\| \|$ & $\|$ & II & $\|$ & $\|$ & ॥ & $\|$ & $\|$ & ॥ & $\|$ & $\|$ & $\begin{array}{l}\text { Reference, } \\
\text { Type II, } \\
\text { ToxoDB\#1 }\end{array}$ \\
\hline $\mathrm{CTG}$ & Cat & & United States & $\|/\| \|$ & III & III & III & III & III & III & III & III & III & III & $\begin{array}{l}\text { Reference, } \\
\text { Type III, } \\
\text { ToxoDB\#2 }\end{array}$ \\
\hline MAS & Human & & France & $\mathrm{u}-1$ & I & $\|$ & III & III & III & $u-1$ & I & I & III & I & $\begin{array}{l}\text { Reference, } \\
\text { ToxoDB\#17 }\end{array}$ \\
\hline TgCgCa1 & Cougar & & Canada & I & । & $\|$ & III & III & $\|$ & $\|$ & $\mathrm{u}-1$ & । & $u-2$ & I & $\begin{array}{l}\text { Reference, } \\
\text { ToxoDB\#66 }\end{array}$ \\
\hline TgCatBr5 & Cat & & Brazil & I & III & III & III & $\|$ & III & 1 & I & I & $\mathrm{u}-1$ & I & $\begin{array}{l}\text { Reference, } \\
\text { ToxoDB\#19 }\end{array}$ \\
\hline TgWtdSc40 & WTD & & United States & $\mathrm{u}-1$ & $\|$ & ॥ & $\|$ & $\|$ & ॥ & $\|$ & $\|$ & I & $\|$ & I & $\begin{array}{l}\text { Reference, } \\
\text { Type } 12, \\
\text { ToxoDB\#5 }\end{array}$ \\
\hline TgCatBr64 & Cat & & Brazil & I & I & $u-1$ & III & III & III & $u-1$ & I & III & III & I & $\begin{array}{l}\text { Reference, } \\
\text { ToxoDB\#111 }\end{array}$ \\
\hline TgRsCr1 & Toucan & & Costa Rica & $\mathrm{u}-1$ & I & II & III & I & III & $u-2$ & I & I & III & I & $\begin{array}{l}\text { Reference, } \\
\text { ToxoDB\#52 }\end{array}$ \\
\hline TgGYn1 & $B G$ & LN & Chuxiong, Yn & I & I & I & I & I & 1 & I & I & I & 1 & I & $\begin{array}{l}\text { Type I, } \\
\text { ToxoDB\#10 }\end{array}$ \\
\hline TgGYn2 & $B G$ & Lung & Qujing, Yn & 1 & । & I & I & I & 1 & I & I & I & I & I & $\begin{array}{l}\text { Type I, } \\
\text { ToxoDB\#10 }\end{array}$ \\
\hline TgGYn3 & $B G$ & LN & Qujing, Yn & I & I & I & I & I & 1 & 1 & I & 1 & nd & I & $\begin{array}{l}\text { Type I, } \\
\text { ToxoDB\#10 }\end{array}$ \\
\hline TgGYn4 & $B G$ & Lung & Qujing, Yn & 1 & I & I & I & 1 & 1 & 1 & I & I & nd & I & $\begin{array}{l}\text { Type I, } \\
\text { ToxoDB\#10 }\end{array}$ \\
\hline TgGYn5 & BG & Liver & Qujing, Yn & I & I & I & 1 & I & 1 & 1 & I & I & nd & I & $\begin{array}{l}\text { Type I, } \\
\text { ToxoDB\#10 }\end{array}$ \\
\hline TgGYn6 & $B G$ & Liver & Qujing, Yn & I & I & I & I & I & I & 1 & I & I & nd & 1 & $\begin{array}{l}\text { Type I, } \\
\text { ToxoDB\#10 }\end{array}$ \\
\hline TgGYn7 & $B G$ & Liver & Kunming, Yn & I & I & I & I & nd & I & 1 & nd & I & I & I & $\begin{array}{l}\text { Type I, } \\
\text { ToxoDB\#10 }\end{array}$ \\
\hline TgGYn8 & $B G$ & LN & Yuxi, Yn & $u-1$ & $\|$ & \| & III & III & ॥ & $\|$ & nd & ॥ & $\|$ & I & ToxoDB\#9 \\
\hline
\end{tabular}

$\mathrm{u}-1$ and $\mathrm{u}-2$ represent unique RFLP genotypes, respectively.

nd: no data. WTD: White-tailed Deer. BG: black goat. LN: Lymph nodes. Yn: Yunnan province.

South America [26-29], as well as other Asian countries, such as Sri Lanka and Vietnam [30,31], indicating that it has a worldwide distribution.

In the present study, another genotype ToxoDB\#10 (Type I) was identified from Yunnan black goats in Qujing, Chuxiong and Kunming of Yunnan province. Previously, this type was also found from humans in Shanghai city, Yunnan and Guangdong province [10,23,24], from cats in Yunnan province [12], from pigs in Hunan, Jiangsu and Henan provinces $[17,24]$, and from bats in Yunnan and Guangxi [13], indicating that ToxoDB\#10 is also a major lineage prevalent in Mainland China.
In this study, limited genetic diversity of $T$. gondii was found in black goats in Yunnan province. A relatively high frequency of the Type I strains found in black goats in this region is of interest. Since the Type I strain is highly virulent to mice and possibly more virulent to humans, future study to isolate viable parasite from this animal is necessary to confirm the finding.

\section{Conclusions}

In summary, the present study revealed an overall prevalence of $4.96 \%$ T. gondii infection in Yunnan black goats by semi-nested PCR of B1 gene, and reported two T. gondii 
genotypes (ToxoDB\#9 and Type I). To our knowledge, this is the first report of these genotypes in Yunnan black goats in China, which may have important implications for public health.

\section{Competing interests}

The authors declare that they have no competing interests.

\section{Authors' contributions}

FCZ and XQZ conceived and designed the study, and critically revised the manuscript. QM, SYH and SYQ performed the experiments, analyzed the data and drafted the manuscript. XY, YY and JFY helped in study design, study implementation and manuscript revision. All authors read and approved the final manuscript.

\section{Acknowledgements}

Project support was provided, in part, by the Yunnan Provincial Program for Introducing High-level Scientists (Grant No. 2009CI125), National Natural Science Foundation of China (Grant No. 31228022), and China Postdoctoral Science Foundation project (2012 M511951) and the Open Funds of the State Key Laboratory of Veterinary Etiological Biology (SKLVEB2011KFKT010). Associate Professor Chunlei Su at Department of Microbiology, the University of Tennessee, Knoxville, USA is thanked for comments and suggestions on the draft manuscript.

\section{Author details}

${ }^{1}$ College of Animal Science and Technology, Yunnan Agricultural University, Kunming, Yunnan Province 650201, PR China. ${ }^{2}$ State Key Laboratory of Veterinary Etiological Biology, Key Laboratory of Veterinary Parasitology of Gansu Province, Lanzhou Veterinary Research Institute, Chinese Academy of Agricultural Sciences, Lanzhou, Gansu Province 730046, PR China. ${ }^{3}$ College of Animal Science and Technology, Jilin Agricultural University, Changchun, Jilin Province 130118, PR China.

Received: 3 September 2014 Accepted: 19 January 2015

Published online: 27 January 2015

\section{References}

1. Dubey JP. Toxoplasmosis of animals and humans. 2nd ed. Boca Raton, Florida: CRC Press; 2010. p. 1-313

2. Jones JL, Lopez A, Wilson M, Schulkin J, Gibbs R. Congenital toxoplasmosis: a review. Obstet Gynecol Survey. 2001;56:296-305.

3. Bobić B, Nikolić A, Klun I, Vujanić M, Djurković-Djaković O. Undercooked meat consumption remains the major risk factor for Toxoplasma infection in Serbia. Parassitologia. 2007:49:227-30.

4. Duan G, Tian YM, Li BF, Yang JF, Liu ZL, Yuan FZ, et al. Seroprevalence of Toxoplasma gondii infection in pet dogs in Kunming, Southwest China. Parasit Vectors. 2012;5:118.

5. Zou FC, Sun XT, Xie YJ, Li B, Zhao GH, Duan G, et al. Seroprevalence of Toxoplasma gondii in pigs in southwestern China. Parasitol Int. 2009:58:306-7.

6. Miao Q, Wang X, She LN, Fan YT, Yuan FZ, Yang JF, et al. Seroprevalence of Toxoplasma gondii in horses and donkeys in Yunnan Province, Southwestern China. Parasit Vectors. 2013;6:168.

7. Tian YM, Dai FY, Huang SY, Deng ZH, Duan G, Zhou DH, et al. First report of Toxoplasma gondii seroprevalence in peafowls in Yunnan Province, Southwestern China. Parasit Vectors. 2012;5:205.

8. Miao Q, Han JQ, Xiang X, Yuan FZ, Liu YZ, Duan G, et al. Prevalence of antibody to Toxoplasma gondii in Black-headed Gulls (Chroicocephalus ridibundus), Dianchi Lake, China. J Wildl Dis. 2014:50:717-9.

9. Ou YX, Xu C, Xue T, Yang YL, Lv R, Song HL, et al. Serological investigation of Toxoplasmosis in goats in Yuxi city. Yunnan J Anin Sci Vet Med. 2014;3:12-3. In Chinese.

10. Nie DP, Jia YX, Chen LJ, You YX, Li W, Shen LJ. Genotypes of Toxoplasma gondii isolates from HIV positive patients in Yunnan Province. Chin J Parasitol Parasit Dis. 2013:31:410-1. In Chinese.

11. Zhou P, Sun XT, Yin CC, Yang JF, Yuan ZG, Yan HK, et al. Genetic characterization of Toxoplasma gondii isolates from pigs in southwestern China. J Parasitol. 2011;97:1193-5.
12. Tian YM, Huang SY, Miao Q, Jiang HH, Yang JF, Su C, et al. Genetic characterization of Toxoplasma gondii from cats in Yunnan Province, Southwestern China. Parasit Vectors. 2014;7:178.

13. Jiang HH, Qin SY, Wang W, He B, Hu TS, Wu JM, et al. Prevalence and genetic characterization of Toxoplasma gondii infection in bats in southern China. Vet Parasitol. 2014;203:318-21.

14. Hill DE, Chirukandoth S, Dubey JP, Lunney JK, Gamble HR. Comparison of detection methods for Toxoplasma gondii in naturally and experimentally infected swine. Vet Parasitol. 2006;141:9-17.

15. Su C, Shwab EK, Zhou P, Zhu XQ, Dubey JP. Moving towards an integrated approach to molecular detection and identification of Toxoplasma gondii. Parasitology. 2010;137:1-11.

16. Jiang HH, Huang SY, Zhou DH, Zhang XX, Su C, Deng SZ, et al. Genetic characterization of Toxoplasma gondii from pigs from different localities in China by PCR-RFLP. Parasit Vectors. 2013;6:227.

17. Zhou P, Nie H, Zhang LX, Wang HY, Yin CC, Su C, et al. Genetic characterization of Toxoplasma gondii isolates from pigs in China. J Parasitol. 2010;96:1027-9.

18. Zhang XX, Huang SY, Zhang YG, Zhang Y, Zhu XQ, Liu Q. First report of genotyping of Toxoplasma gondii in free-living Microtus fortis in Northeastern China. J Parasitol. 2014;100:692-4.

19. Chen ZW, Gao JM, Huo XX, Wang L, Yu L, Halm-Lai F, et al. Genotyping of Toxoplasma gondii isolates from cats in different geographic regions of China. Vet Parasitol. 2011;183:166-70.

20. Wang L, Cheng HW, Huang KQ, Xu YH, Li YN, Du J, et al. Toxoplasma gondii prevalence in food animals and rodents in different regions of China: isolation, genotyping and mouse pathogenicity. Parasit Vectors. 2013;6:273.

21. Qian W, Wang H, Su C, Shan D, Cui X, Yang N, et al. Isolation and characterization of Toxoplasma gondii strains from stray cats revealed a single genotype in Beijing, China. Vet Parasitol. 2012;187:408-13.

22. Dubey JP, Zhu XQ, Sundar N, Zhang H, Kwok OC, Su C. Genetic and biologic characterization of Toxoplasma gondii isolates of cats from China. Vet Parasitol. 2007;145:352-6.

23. Wang L, Chen H, Liu D, Huo X, Gao J, Song X, et al. Genotypes and mouse virulence of Toxoplasma gondii isolates from animals and humans in China. PLOS ONE. 2013;8:e53483.

24. Zhou P, Zhang H, Lin RQ, Zhang DL, Song HQ, Su C, et al. Genetic characterization of Toxoplasma gondii isolates from China. Parasitol Int. 2009;58:193-5.

25. Wang H, Wang T, Luo Q, Huo X, Wang L, Liu T, et al. Prevalence and genotypes of Toxoplasma gondii in pork from retail meat stores in Eastern China. Int J Food Microbiol. 2012;157:393-7.

26. Dubey JP, Sundar N, Hill D, Velmurugan GV, Bandini LA, Kwok OC, et al. High prevalence and abundant atypical genotypes of Toxoplasma gondil isolated from lambs destined for human consumption in the USA. Int J Parasitol. 2008:38:999-1006.

27. Dubey JP, Velmurugan GV, Alvarado-Esquivel C, Alvarado-Esquivel D, Rodriguez-Pena S, Martinez-Garcia S, et al. Isolation of Toxoplasma gondii from animals in Durango, Mexico. J Parasitol. 2009;95:319-22.

28. Dubey JP, Cortes-Vecino JA, Vargas-Duarte JJ, Sundar N, Velmurugan GV, Bandini LM, et al. Prevalence of Toxoplasma gondii in dogs from Colombia, South America and genetic characterization of $T$. gondii isolates. Vet Parasitol. 2007;145:45-50.

29. Dubey JP, Sundar N, Gennari SM, Minervino AH, Farias NA, Ruas JL, et al. Biologic and genetic comparison of Toxoplasma gondii isolates in free-range chickens from the northern Para state and the southern state Rio Grande do Sul, Brazil revealed highly diverse and distinct parasite populations. Vet Parasitol. 2007;143:182-8.

30. Dubey JP, Huong LT, Sundar N, Su C. Genetic characterization of Toxoplasma gondii isolates in dogs from Vietnam suggests their South American origin. Vet Parasitol. 2007;146:347-51.

31. Dubey JP, Rajapakse RP, Wijesundera RR, Sundar N, Velmurugan GV, Kwok OC, et al. Prevalence of Toxoplasma gondii in dogs from Sri Lanka and genetic characterization of the parasite isolates. Vet Parasitol. 2007;146:341-6. 